PROCEEDINGS OF THE

AMERICAN MATHEMATICAL SOCIETY

Volume 128, Number 11, Pages 3211-3219

S 0002-9939(00)05550-7

Article electronically published on May 11, 2000

\title{
DEGREES, KERNELS AND QUASIKERNELS OF MONOLITHIC CHARACTERS
}

\author{
YAKOV BERKOVICH
}

(Communicated by Ronald M. Solomon)

\begin{abstract}
Theorem 5 yields the condition sufficient for a group to be a direct product of a $\pi$-group and an abelian $\pi^{\prime}$-group. We also obtain characterizations of nilpotent groups, prime power groups, $p$-nilpotent and $p$-closed groups in the language of characters. Proofs of some results depend on the classification of finite simple groups. Some problems are posed and discussed.
\end{abstract}

Only finite groups are considered. Let $\pi, \pi^{\prime}$ be complementary sets of primes. A group $G$ is said to be $\pi$-nilpotent $\left(\pi\right.$-closed) if the set of all its $\pi^{\prime}$-elements $(\pi$ elements) is a subgroup of $G$. A group is $\pi$-closed if and only if it is $\pi^{\prime}$-nilpotent. Let $\Phi(G)$ be the Frattini subgroup of $G$. In what follows, we use the following known facts (see, for example, [BZ], Lemma 14.1(a), (c)):

( $\Phi 1) G / \Phi(G)$ is $\pi$-closed if and only if $G$ is.

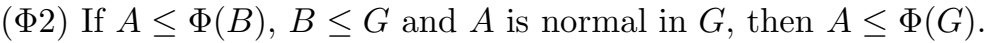

Let $\pi(m)$ be the set of prime divisors of $m \in \mathbb{N}, \pi(G)=\pi(|G|)$. For $m \in \mathbb{N}, m_{\pi}$ denotes the maximal $\pi$-divisor of $m$. Let $\operatorname{Irr}(G)$ be the set of (complex) irreducible characters of $G$. If $\chi \in \operatorname{Irr}(G)$, then the subset $\mathrm{Z}(\chi)=\{x \in G|| \chi(x) \mid=\chi(1)\}$ is a normal subgroup of $G$ - the quasikernel of $\chi$. In that case, $\mathrm{Z}(\chi) / \operatorname{ker}(\chi)=$ $\mathrm{Z}(G / \operatorname{ker}(\chi))$ is cyclic.

Let $\operatorname{Syl}_{p}(G)$ be the set of Sylow $p$-subgroups of $G, p$ a prime. If $K$ is a normal subgroup of $G$, then $\operatorname{Irr}(G \mid K)$ is the set of $\chi \in \operatorname{Irr}(G)$ such that $K \not \leq \operatorname{ker}(\chi)$.

Recall that a character $\chi$ of $G$ is said to be monolithic if $\chi$ is irreducible and $G / \operatorname{ker}(\chi)$ is a monolith, i.e., has only one minimal normal subgroup (see [BZ], Chapter 30, and [BIK]). Note that every irreducible character of a $p$-group is monolithic.

It is known that the degree of an irreducible character $\chi$ of $G$ divides $|G / \mathrm{Z}(\chi)|$. So we may assume that the structure of $G$ depends essentially on quotients $|G| / \chi(1)$, $|G / \operatorname{ker}(\chi)| / \chi(1)$ and $|G / \mathrm{Z}(\chi)| / \chi(1)$ for some nonlinear $\chi \in \operatorname{Irr}(G)$.

Chillag and Herzog have classified the nonabelian groups $G$ such that $|G| / \chi(1)$ is a prime power for every nonlinear $\chi \in \operatorname{Irr}(G)$ (see [BZ], Theorem 14.31). It appears that then $|G| / \chi(1) \mid$ is a power of the prime independent of $\chi$, and $G$ is solvable of very special structure. In the following lemma we consider a more general situation.

Received by the editors January 21, 1999.

1991 Mathematics Subject Classification. Primary 20C15.

Key words and phrases. $\pi$-closed and $\pi$-nilpotent groups, kernel and quasikernel, monolithic character, Frobenius group, classification of finite simple groups.

The author was supported in part by the Ministry of Absorption of Israel. 
Lemma 1 (Compare to [BZ], Exercise 14.25). Let $\chi \in \operatorname{Irr}(G)$. Suppose that $G / \operatorname{ker}(\chi)$ has a normal abelian subgroup $A / \operatorname{ker}(\chi)$ such that $|G: A|=p^{s} \cdot \chi(1)$ for some prime power $p^{s}$. Then $\chi$ is monomial. In particular, if the above holds for all nonlinear monolithic characters $\chi$ of $G$ with $p^{s}$ depending on $\chi$, then $G$ is solvable.

Proof. Note that $\chi(1)$ divides $|G: A|$, where $\chi$ and $A$ as in the lemma, by $[$, Theorem 6.15 (Ito's Theorem). By [BZ], Proposition 30.18(e), the second assertion follows from the first one. To prove that $\chi$ is monomial, one may assume that $\operatorname{ker}(\chi)=\{1\}$. Set $H=P A$, where $P \in \operatorname{Syl}_{p}(G)$. Let $\left\{\mu_{1}, \ldots, \mu_{k}\right\}$ be the set of irreducible constituents of $\chi_{H}, \mu_{1}(1) \leq \cdots \leq \mu_{k}(1)$. By Ito's Theorem, $\mu_{i}(1)$ are powers of $p$ for all $i$. It follows that $\mu_{1}(1)$ divides $\chi(1)$. Furthermore, $|G: H|$ divides $\chi(1)$ by hypothesis, and $\left(|G: H|, \mu_{1}(1)\right)=1$. It follows that $|G: H| \mu_{1}(1)=\mu_{1}^{G}(1)$ also divides $\chi(1)$. Therefore, by reciprocity, $\chi=\mu_{1}^{G}$. By Huppert's monomiality criterion (see [BZ], Theorem 7.61), $H$ is an $M$-group. Therefore, $\mu_{1}=\lambda^{H}$ for some $F \leq H$ and a linear character $\lambda$ of $F$. Thus $\chi=\left(\mu_{1}\right)^{G}=\left(\lambda^{H}\right)^{G}=\lambda^{G}$, completing the proof.

We will define some characteristic subgroups of $G$.

Let $G\left(p^{\prime}\right)\left(G_{0}\left(p^{\prime}\right)\right)$ be the intersection of kernels (quasikernels) of nonlinear irreducible characters of $G$ having $p^{\prime}$-degrees. Obviously, $G\left(p^{\prime}\right) \leq G_{0}\left(p^{\prime}\right)$. By [BZ], Remark 25.2, if $P \in \operatorname{Syl}_{p}(G)$, then $p$ divides degrees of all nonlinear irreducible characters of $P G\left(p^{\prime}\right)$. In particular, $G\left(p^{\prime}\right)$ is $p$-nilpotent and solvable (see $\mathbb{I}$, Corollary 12.2, and [BZ], Proposition 25.9 and the remark following it).

Let $G(p)\left(G_{0}(p)\right)$ be the intersection of kernels (quasikernels) of irreducible characters of $G$ whose degrees are divisible by $p$. It is known that $G(p)$ is $p$-closed (see [BZ, Theorem 14.27(c)); obviously, $G(p) \leq G_{0}(p)$. By [BZ, Remark 25.3, all nonlinear irreducible characters of $G(p)$ have $p^{\prime}$-degrees so $G(p)$ is $p$-closed with abelian Sylow $p$-subgroup. It is known that $G_{0}\left(p^{\prime}\right) \cap G_{0}(p)=\mathrm{Z}(G)$ (see [I] , Corollary 2.28, or [BZ, Theorem 4.35(b)).

It is known (see $[\mathrm{B}$, Lemma 2(b)) that the intersection of kernels of nonlinear monolithic characters of $G$ is contained in $\mathrm{Z}(G)$ (the strong containment is possible: let $G$ be a nonabelian primary group with cyclic center). Let $p$ be a prime divisor of $|G|$. Set $G_{m}\left(p^{\prime}\right)=\bigcap_{\chi} \operatorname{ker}(\chi)$, where $\chi$ runs over all nonlinear monolithic characters of $G$ of $p^{\prime}$-degrees. Obviously, $G\left(p^{\prime}\right) \leq G_{m}\left(p^{\prime}\right)$.

Let $G_{m}(p)$ be the intersection of kernels of monolithic characters of $G$, whose degrees are divisible by $p$; obviously, $G(p) \leq G_{m}(p)$. By what has been said already, $G_{m}\left(p^{\prime}\right) \cap G_{m}(p) \leq \mathrm{Z}(G)$.

In Theorem 2 we will consider the structure of the subgroups introduced above.

Theorem 2. Let $p$ be a prime divisor of the order of a group $G, P \in \operatorname{Syl}_{p}(G)$. Then

(a) All nonlinear irreducible characters of $P G_{0}\left(p^{\prime}\right)$ have degrees divisible by $p$; in particular, $G_{0}\left(p^{\prime}\right)$ is p-nilpotent and solvable.

(b) $G_{m}\left(p^{\prime}\right)$ is p-nilpotent and solvable.

(c) All nonlinear irreducible characters of $G_{0}(p)$ have $p^{\prime}$-degrees; in particular,

$G_{0}(p)$ is p-closed with abelian Sylow p-subgroup.

(d) $G_{m}(p)$ is p-closed.

Proof. Suppose, in all the cases, that $G$ is a counterexample of minimal order.

(a) Let $\lambda$ be a nonlinear irreducible character of $P G_{0}\left(p^{\prime}\right)$ of $p^{\prime}$-degree. Then $p$ does not divide $\lambda^{G}(1)=\left|G: P G_{0}\left(p^{\prime}\right)\right| \lambda(1)$ so $\lambda^{G}$ has an irreducible constituent 
$\chi$ of $p^{\prime}$-degree. By reciprocity, $\chi$ is nonlinear so, by hypothesis, $G_{0}\left(p^{\prime}\right) \leq \mathrm{Z}(\chi)$. All irreducible constituents of $\chi_{\mathrm{Z}(\chi)}$ are linear and the same is true for $\lambda_{G_{0}\left(p^{\prime}\right)}$. It follows that $G_{0}\left(p^{\prime}\right)^{\prime} \leq \operatorname{ker}(\lambda)$, by Clifford's Theorem. Since $P G_{0}\left(p^{\prime}\right) / G_{0}\left(p^{\prime}\right)^{\prime}$ is an extension of the abelian group $G_{0}\left(p^{\prime}\right) / G_{0}\left(p^{\prime}\right)^{\prime}$ by the $p$-group $P G_{0}\left(p^{\prime}\right) / G_{0}\left(p^{\prime}\right)$, it follows that $\lambda(1)$ is a power of $p$, by Ito's Theorem, which is a contradiction. Thus, degrees of all nonlinear irreducible characters of $P G_{0}\left(p^{\prime}\right)$ are divisible by $p$ so $P G_{0}\left(p^{\prime}\right)$ is $p$-nilpotent by [I], Corollary 12.2 , and solvable by [BZ], Proposition 25.9 and the remark following it. ((a) generalizes [BZ], Remark 25.2.)

(b) Write $D=G_{m}\left(p^{\prime}\right)$. We may assume that $D>\{1\}$. Let $K$ be a minimal normal subgroup of $G$ contained in $D$. By the inductive hypothesis, $D / K$ is $p$ nilpotent and solvable (indeed, $\left.(G / K)_{m}\left(p^{\prime}\right)=D / K\right)$ so $p$ divides $|K|$. Assume that $K_{1}$ is another minimal normal subgroup of $G$ contained in $D$; then $D / K_{1}$ is $p$ nilpotent and solvable. Since $K \cap K_{1}=\{1\}, D$ is also $p$-nilpotent and solvable, and $G$ is not a counterexample. Thus, $K$ is the unique minimal normal subgroup of $G$ contained in $D$. Let $M$ be a normal subgroup of $G$ maximal such that $K \cap M=\{1\}$. By what we just proved, $D \cap M=\{1\}$. Assume that $M>\{1\}$. As $D M / M \leq$ $(G / M)_{m}\left(p^{\prime}\right)$ and $D M / M \cong D$ is $p$-nilpotent, by the inductive hypothesis, we obtain a contradiction. Thus $M=\{1\}$. In that case, $G$ is a monolith.

Let $\chi \in \operatorname{Irr}(G \mid K)$. Since $K \leq G^{\prime}$ as the unique minimal normal subgroup of $G, \chi$ is nonlinear monolithic. We have $D \not \leq \operatorname{ker}(\chi)=\{1\}$ so $p$ divides $\chi(1)$. It follows that $K \leq G\left(p^{\prime}\right)$ so $K$ is solvable (see the text preceding the theorem). Since $p$ divides $|K|$, we see that $K$ is a $p$-subgroup and so $D$ is solvable. By the above, $D / K$ has the normal $p$-complement $F / K$. Since $D$ is not a $p$-subgroup and $G$ is a monolith, we have $F>K$ and $\mathrm{O}_{p^{\prime}}(F)=\{1\}$. Let $P \in \operatorname{Syl}_{p}(G)$. Then, $K \not \leq \Phi(P)$ (otherwise, $K \leq \Phi(G)$, by $(\Phi 2)$, and $D$ is $p$-nilpotent, by $(\Phi 1)$ ). In that case, there is a linear character $\lambda \in \operatorname{Irr}(P \mid K)$. Since $p$ does not divide $\lambda^{G}(1)=|G: P|$, $\lambda^{G}$ has an irreducible constituent $\chi$ of $p^{\prime}$-degree. On the other hand, $K \not \leq \operatorname{ker}(\chi)$, by reciprocity so $\chi$ is nonlinear monolithic (recall that $K \leq G^{\prime}$ ). It follows from $D \not \operatorname{ker}(\chi)$ that $\chi$ is of degree divisible by $p$, which is a contradiction. (This generalizes [BZ], Proposition 30.18(b).)

(c) Let $\lambda$ be an irreducible character of $G_{0}(p)$ of degree divisible by $p$ and let $\chi$ be an irreducible constituent of $\lambda^{G}$. Since $p$ divides $\chi(1)$, by Clifford's Theorem, we get $G_{0}(p) \leq \mathrm{Z}(\chi)$, by definition. As all irreducible constituents of $\chi_{\mathrm{Z}(\chi)}$ are linear, the same is true for $\chi_{G_{0}(p)}$. Since $\lambda$ is nonlinear, we get a contradiction. Thus, all nonlinear irreducible characters of $G_{0}(p)$ have $p^{\prime}$-degrees so $G_{0}(p)$ is $p$-closed with abelian Sylow $p$-subgroup, by Ito-Michler Theorem for $p$-solvable groups ( $\mathbb{I}$, Corollary 12.34, is also true for $p$-solvable groups). ((c) generalizes [BZ], Remark 25.3.)

(d) Write $D=G_{m}(p)$. Let $K$ be a minimal normal subgroup of $G$ contained in $D$. By the inductive hypothesis, $D / K$ is $p$-closed. As in (b), $K$ is the unique minimal normal subgroup of $G$ contained in $D$. Let $M$ be a normal subgroup of $G$ maximal such that $K \cap M=\{1\}$. Since $D \cap M=\{1\}$ (so $D M / M \cong D$ ) and $D M / M \leq(G / M)_{m}(p)$, we see that $M=\{1\}$, by the inductive hypothesis. It follows that $G$ is a monolith so it possesses a faithful irreducible character $\chi$; obviously, $\chi$ is nonlinear monolithic. Since $D \not \leq \operatorname{ker}(\chi), \chi$ is of $p^{\prime}$-degree. It follows that $K \leq G(p)$ so $K$ is $p$-closed (see the text preceding the theorem). As $D$ is not $p$-closed, $K$ is a $p^{\prime}$-subgroup so $\mathrm{O}_{p}(D)=\{1\}$ since $G$ is a monolith.

Let $F / K$ be a minimal normal $p$-subgroup of $G / K$ contained in $D / K$; then $F / K>\{1\}$ is an elementary abelian $p$-subgroup (recall that $D / K$ is $p$-closed and 
$p$ divides $|D / K|)$. Then $\mathrm{O}_{p}(F)=\{1\}$ since $G$ is a monolith. By the Ito-Michler Theorem, $F$ has a nonlinear irreducible character $\lambda$ of degree divisible by $p$. Since $F / K$ is abelian, we have $K \not \leq \operatorname{ker}(\lambda)$. Let $\chi$ be an irreducible constituent of $\lambda^{G}$. By reciprocity, $\chi$ is monolithic, and $p$ divides $\chi(1)$, by Clifford's Theorem. Then $K \leq D \leq \operatorname{ker}(\chi)$, by definition, which is a contradiction. Thus there is no counterexample and so $D$ is $p$-closed. ((d) generalizes the main part of [BZ], Proposition 30.18(c).)

Proposition 3. Let $p$ be a prime divisor of the order of a group $G$.

(a) $G$ is p-nilpotent if and only if for every nonlinear monolithic character $\chi$ of $G$ such that $p$ does not divide $\chi(1), G / \operatorname{ker}(\chi)$ is p-nilpotent.

(b) $G$ is $p$-closed if and only if for every nonlinear monolithic character $\chi$ of $G$ such that $p$ divides $\chi(1), G / \operatorname{ker}(\chi)$ is $p$-closed.

Proof. Suppose that $G$ is a counterexample of minimal order. Then it has only one minimal normal subgroup $K$.

(a) In that case, $G / K$ is $p$-nilpotent. Let $\chi$ be a nonlinear monolithic character of $G$ of $p^{\prime}$-degree (if such a $\chi$ does not exist, we have $G=G_{m}\left(p^{\prime}\right)$, and this group is $p$-nilpotent and solvable, by Theorem $2(\mathrm{~b}))$. By hypothesis, $G / \operatorname{ker}(\chi)$ is $p$ nilpotent. Then $G / G_{m}\left(p^{\prime}\right)$ is $p$-nilpotent as an epimorphic image of $G / \operatorname{ker}(\chi)$ so $\{1\}<G_{m}\left(p^{\prime}\right)<G, K \leq G_{m}\left(p^{\prime}\right)$ and $K$ is solvable, by Theorem 2(b). It follows that $K$ is a $p$-subgroup. Let $P \in \operatorname{Syl}_{p}(G)$; then $K \leq P$ and $K \not \leq \Phi(P)$ (see the proof of Theorem 2(b)). Repeating, word for word, the last rows of the proof of Theorem 2(b), we complete the proof of (a).

(b) In that case, $G / K$ is $p$-closed. Let $\chi$ be a nonlinear monolithic character of $G$ of degree divisible by $p$ (if such a $\chi$ does not exist, we have $G=G_{m}(p)$, and this group is $p$-closed, by Theorem $2(\mathrm{~d}))$. By hypothesis, $G / \operatorname{ker}(\chi)$ is $p$-closed. It follows that $G / G_{m}(p)$ is $p$-closed as an epimorphic image of $G / \operatorname{ker}(\chi)$ so $\{1\}<G_{m}(p)<G$; thus $K \leq G_{m}(p)$ and $K$ is $p$-closed. It follows that $K$ is a $p^{\prime}$-subgroup. Let $F / K$ be a minimal normal $p$-subgroup of $G / K$ contained in $D / K$; then $F / K>\{1\}$ is elementary abelian. As $G$ is a monolith, $\mathrm{O}_{p}(F)=\{1\}$. Repeating, word for word, the last rows of the proof of Theorem 2(d), we complete the proof of (b).

It follows from Proposition 3(a) that $G$ is nilpotent if and only if $\pi(G / \mathrm{Z}(\chi))=$ $\pi(\chi(1))$ for every monolithic character $\chi$ of $G$. Indeed, let $p \in \pi(G)$ and $p$ does not divide $\chi(1)$. Then $G / \mathrm{Z}(\chi)$ is a $p^{\prime}$-group, by assumption. It then follows that $G / \operatorname{ker}(\chi)$ is $p$-nilpotent, and our claim is true, by Proposition 3(a) since $p$ is arbitrary. Next, a nonabelian group $G$ is nilpotent if and only if $\pi(G / \operatorname{ker}(\chi))=\pi(\chi(1))$ for every nonlinear monolithic character $\chi$ of $G$ (the same reasoning!). If the last condition holds for all nonlinear $\chi \in \operatorname{Irr}(G)$, then $G$ is a prime power group as it is easy to check.

Assertions (a), (b) of Proposition 3 characterize $p$-nilpotent and $p$-closed groups, respectively.

Lemma 4 (Compare to [BZ], Theorem 14.45). If $\pi(G / Z(\chi)) \subseteq \pi \cup \pi(\chi(1))$ for every monolithic character $\chi$ of $G$, then $G$ is $\pi$-closed. Furthermore, if $P$ is a $\pi$-Hall subgroup of $G$, then $G / P$ is nilpotent.

Proof. Let $G$ be a $\pi^{\prime}$-group. Then by hypothesis, $\pi(G / \mathrm{Z}(\chi)) \subseteq \pi(\chi(1))$ for every monolithic character $\chi$ of $G$; the converse inclusion follows from Ito's Theorem. So $G$ is nilpotent, by the remark preceding the lemma. Therefore, we may assume that $\pi \cap \pi(G)$ is nonempty. 
Suppose that $G$ is a counterexample of minimal order. Let $H$ be a minimal normal subgroup of $G$. By the inductive hypothesis, $G / H$ is $\pi$-closed. It follows that $H$ is the unique minimal normal subgroup of $G$. Assume that $\mathrm{Z}(G)>\{1\}$. Then $H \leq \mathrm{Z}(G)$, and since $G / H$ is $\pi$-closed so is $G$, a contradiction. Thus $\mathrm{Z}(G)=\{1\}$. Since $H$ is unique, $G$ possesses a faithful irreducible character $\chi$; obviously, $\chi$ is monolithic and $\mathrm{Z}(\chi)=\{1\}$. Then by hypothesis, $\pi(G) \subseteq \pi \cup \pi(\chi(1))$, and we see that $\chi(1)$ is divisible by all $q \in \pi(G) \cap \pi^{\prime}$. Since $\chi$ is an arbitrary faithful irreducible character of $G$, we conclude that $H \leq G\left(q^{\prime}\right)$ for every $q \in \pi(G) \cap \pi^{\prime}$ so $H$ is solvable, by Theorem 2(b). Thus, $H$ is a $p$-group for some $p \in \pi^{\prime}$ since $G / H$ is $\pi$-closed and $G$ is not.

Let $H \leq P \in \operatorname{Syl}_{p}(G)$. If $H \leq \Phi(P)$, then $H \leq \Phi(G)$, by ( $\left.\Phi 2\right)$, and $G$ is $\pi$-closed since $G / H$ is, by $(\Phi 1)$. Thus $H \nless \leq \Phi(P)$; then $H \not P^{\prime}$. In that case, there is a linear character $\lambda$ of $P$ such that $H \not \leq \operatorname{ker}(\lambda)$. Every irreducible constituent of $\lambda^{G}$ is faithful, by reciprocity so monolithic. Since $p$ does not divide $\lambda^{G}(1)=|G: P|$, there is an irreducible constituent $\chi$ of $\lambda^{G}$ of $p^{\prime}$-degree. Then $\pi(G) \nsubseteq \nsubseteq \pi \cup \pi(\chi(1))$ since $p \notin \pi \cup \pi(\chi(1))$, which is a contradiction. The last assertion follows from the first paragraph of the proof.

If $\chi$ is a nonlinear irreducible character of $G$, then $|G / \operatorname{ker}(\chi)|=t_{\chi} \cdot \chi(1)$ for some $t_{\chi} \in \mathbb{N}$. It is possible that $\pi(G) \neq \bigcup_{\chi \in \operatorname{Irr}(G), \chi(1)>1} \pi\left(t_{\chi}\right)$. As Theorem $5(\mathrm{c})$ shows, in that case $G$ has very special structure (in particular, it is solvable).

In Theorem $5\left(\right.$ a) we will consider a special case when $\chi(1)^{2}$ divides $|G|$ for all $\chi \in \operatorname{Irr}(G)$. It is interesting that the set of groups satisfying this property is very large: every nonabelian group is a direct factor of such a group (see the Remark below). I think that if $G>\{1\}$ satisfies the above condition, then its Fitting subgroup is nontrivial. Note that $\chi(1)^{2}$ divides $|G / \operatorname{ker}(\chi)|$ for all irreducible characters $\chi$ of every nilpotent group $G$ (it is remarkable that the converse is also true; see the Remark preceding Corollary 6 ). Corollary 6 characterizes nonabelian $p$-groups. If there is $t_{\chi} \in \mathbb{N}$ such that $|G|=t_{\chi} \cdot \chi(1)^{2}$ for every nonlinear monolithic character $\chi$ of $G$, then $G$ is $\pi$-closed, where $\pi=\bigcup_{\chi \in \operatorname{Irr}(G), \chi(1)>1} \pi\left(t_{\chi}\right)$, by Lemma 4 .

Let us consider, for $\chi \in \operatorname{Irr}(G)$, the following condition:

$$
|G / \operatorname{ker}(\chi)|=t \cdot \chi(1)^{s} \text { for some natural } s=s_{\chi} \text { and } t=t_{\chi} .
$$

Theorem 5. Let $G$ be a nonabelian group.

(a) Suppose that every nonlinear monolithic character $\chi$ of $G$ satisfies $(*)$ with $s_{\chi}=2$. Set $\pi=\bigcup_{\chi} \pi\left(t_{\chi}\right)$, where $\chi$ runs over all nonlinear monolithic characters of $G$. Then $G=P \times A$, where $A$ is an abelian $\pi^{\prime}$-Hall subgroup of $G$.

(b) Suppose that every nonlinear irreducible character $\chi$ of $G$ satisfies $(*)$ with $s_{\chi}=2$. Set $\pi=\bigcup_{\chi \in \operatorname{Irr}(G), \chi(1)>1} \pi\left(t_{\chi}\right)$. Then $\pi(G)=\pi$.

(c) Suppose that every nonlinear irreducible character $\chi$ of $G$ satisfies $\left(^{*}\right)$ with $s_{\chi}=1$. Let $\pi=\bigcup_{\chi \in \operatorname{Irr}(G), \chi(1)>1} \pi\left(t_{\chi}\right)$. Then $G$ has the normal $\pi$-Hall subgroup $P$ and $G / P$ is cyclic. Furthermore, if $P<G$, then $P$ is solvable. In that case, if $F$ is a $\pi^{\prime}$-Hall subgroup of $G$ and $K$ a normal subgroup of $G$ such that $K<G^{\prime}$ and $G^{\prime} / K$ is a minimal normal subgroup of $G / K$, then every element of $F^{\#}$ induces a fixed-point-free automorphism on $G^{\prime} / K$.

Proof. Assume that $G$ is a $\pi^{\prime}$-group and a constant $s \in\{1,2\}$. Then, for every nonlinear monolithic character $\chi$ of $G$, we have $|G / \operatorname{ker}(\chi)|=\chi(1)^{s}$, which is not 
the case. Since a nonabelian group has a nonlinear monolithic character, $G$ is not a $\pi^{\prime}$-group. By Lemma $4, G$ is $\pi$-closed; let $P$ be a (normal) $\pi$-Hall subgroup of $G$. Since $G / P$ is a $\pi^{\prime}$-group, by what we just proved, it is abelian. Let $G$ be a counterexample of minimal order.

Note that if $G=P \times A$, then by the result of the previous paragraph $A$ is abelian and then in cases (b) and (c) we have $A=\{1\}$, as is easy to check.

(a) Let $s=s_{\chi}=2$ for all nonlinear monolithic characters $\chi$ of $G$, and suppose that $G$ is not a $\pi$-group. Obviously, nonabelian epimorphic images of $G$ satisfy the hypothesis. Let $H$ be a minimal normal subgroup of $G$. By the inductive hypothesis, $G / H=P_{1} / H \times A_{1} / H$, where $A_{1} / H$ is the abelian $\pi^{\prime}$-Hall subgroup of $G / H$. It follows that $H$ is the unique minimal normal subgroup of $G$. Then $H \leq P$ so $H$ is a $\pi$-group. In that case, $G$ possesses a faithful irreducible character $\chi$; obviously, $\chi$ is monolithic. By hypothesis, $|G|=t_{\chi} \cdot \chi(1)^{2}$ so $|G|_{\pi^{\prime}}=\left(\chi(1)^{2}\right)_{\pi^{\prime}}$. Since $\chi$ is arbitrary faithful, it follows that $H \leq G\left(q^{\prime}\right)$ for all $q \in \pi(G)-\pi \neq \emptyset$ so $H$ is solvable, by Theorem 2(a). Thus, $H$ is a $p$-subgroup for some $p \in \pi$. By $(\Phi 1)$ and the result of the second paragraph of the proof, $H \not \leq \Phi(G)$. Therefore, $G=M \cdot H$, a semidirect product, where $M$ is maximal in $G$. We have $M \cong G / H$. Let $A_{0}$ be a $\pi^{\prime}$-Hall subgroup of $M$ (then $A_{0}$ is also a $\pi^{\prime}$-Hall subgroup of $G$ ). Take $x \in A_{0}^{\#}$. Since $H$ is the unique minimal normal subgroup of $G$ and $A_{0}$ is abelian, we get $\mathrm{C}_{G}(x)=M$. It follows that $A_{1}=A_{0} \cdot H$ is a Frobenius group. Let $\lambda$ be a nonlinear irreducible character of $A_{1}$; then $\lambda(1)=\left|A_{0}\right|=|G|_{\pi^{\prime}}$. Let $\theta$ be an irreducible constituent of $\lambda^{G}$. By reciprocity, $\theta$ is monolithic. By (*), $|G|_{\pi^{\prime}}=\left(\theta(1)^{2}\right)_{\pi^{\prime}}$, a contradiction since $|G|_{\pi^{\prime}}>1$ divides $\theta(1)$, by Clifford's Theorem (recall that $A_{1}$ is normal in $G$ ). This proves (a).

(b) follows from (a), by the second paragraph of the proof.

(c) Let $s=s_{\chi}=1$ for all nonlinear $\chi \in \operatorname{Irr}(G)$. By the first paragraph of the proof, $G / P$ is abelian. Assume that $P<G$.

We will prove that $G$ is solvable. Suppose there $G$ has two distinct minimal normal subgroups $H_{1}, H_{2}$. We may assume that $H_{1} \leq P$, i.e., $H_{1}$ is a $\pi$-subgroup. Then by induction, $G / H_{1}$ is solvable. If $G / H_{2}$ is not a $\pi$-group, then $G / H_{2}$ is also solvable. Then $G$ is solvable as a subgroup of $\left(G / H_{1}\right) \times\left(G / H_{2}\right)$. It remains to consider the case when $G / H_{2}$ is a $\pi$-group. Then $\left|H_{2}\right|=p \in \pi^{\prime}$ and $G=P \times H_{2}$, contrary to the second paragraph of the proof. Thus, we may assume that $H$ is the unique minimal normal subgroup of $G$. Then $G$ has a faithful irreducible character $\chi$ so $|G|=t_{\chi} \cdot \chi(1)$. It follows that $|G|_{\pi^{\prime}}$ divides $\chi(1)$ so $H \leq G\left(q^{\prime}\right)$ for every $q \in \pi(G) \cap \pi^{\prime} \neq \emptyset$. By Theorem 2(a), $H$ is solvable. As $G / H$ is solvable so is $G$.

We have $G^{\prime} \leq P$. To prove the remaining assertion, we may assume that $G^{\prime}$ is a minimal normal subgroup of $G$. Then $G^{\prime}$ is a $p$-subgroup for some $p \in \pi$.

By the result of the second paragraph of the proof, $G$ is not nilpotent. Then $G^{\prime} \not \mathbf{Z}(G)$ and $G^{\prime} \not \leq \Phi(G)$ so $G$ has a maximal subgroup $M$ such that $G^{\prime} \not M$; then $G=M \cdot G^{\prime}$, a semidirect product since $G^{\prime}$ is an abelian minimal normal subgroup of $G$. Obviously, $M \cong G / G^{\prime}$ is abelian. Let $F$ be a $\pi^{\prime}$-Hall subgroup of $M$ and let $x \in F^{\#}$ such that its order is a power of a prime $q$. Then $\mathrm{C}_{G}(x) \geq M$ so we have $\mathrm{C}_{G^{\prime}}(x) \unlhd G$. Assume that $\mathrm{C}_{G^{\prime}}(x)=G^{\prime}$. Then $x \in \mathrm{Z}(G)$, and since $\bigcap_{\chi \in \operatorname{Irr}(G), \chi(1)>1} \operatorname{ker}(\chi)=\{1\}$ (see [BZ], Theorem 4.35), there is a nonlinear $\chi \in$ $\operatorname{Irr}(G)$ such that $x \notin \operatorname{ker}(\chi)$. As by Ito's Theorem $\chi(1)$ divides $|G:(\langle x\rangle \times \operatorname{ker}(\chi))|$; altogether with our hypothesis we conclude that

$$
q \cdot|G:(\langle x\rangle \times \operatorname{ker}(\chi))||| G / \operatorname{ker}(\chi)\left|=t_{\chi} \chi(1)\right| t_{\chi} \cdot|G:(\langle x\rangle \times \operatorname{ker}(\chi))|
$$


and thus $q$ divides $t_{\chi}$, contradicting $t_{\chi}$ being a $\pi$-number. So since $G^{\prime}$ is an abelian minimal normal subgroup of $G$, we have $\mathrm{C}_{G^{\prime}}(x)=\{1\}$ and since $x \in F^{\#}$ is an arbitrary of prime power order, we see that $F G^{\prime}$ is a Frobenius group; in particular $F \cong G / P$ is cyclic, and (c) is proven.

In Theorem 5(a), the hypothesis $s_{\chi}=2$ is essential. It is impossible to change that condition by $s_{\chi}>1$ for all nonlinear monolithic characters $\chi$ of $G$ (take, for example, a nonabelian group of order $p^{3}$ and $s_{\chi}=3$ for all nonlinear irreducible $\chi)$.

Remark. Let $G$ be nonabelian. If $\chi(1)^{2}$ divides $|G|$ for all $\chi \in \operatorname{Irr}(G)$, then $G$ need not be solvable: take, for example, $G=H \times \mathrm{C}_{|H|}$, where $\mathrm{C}_{m}$ is the cyclic group of order $m$ and $H$ nonsolvable. It follows from the classification of finite simple groups that $G$ is not simple. (The simple groups of Lie type do not satisfy this condition: by $[\mathrm{W}]$, they have an irreducible character of $p$-defect 0 for every prime $p$. The alternating group $\mathrm{A}_{n}$ does not satisfy that condition since it possesses an irreducible character of degree divisible by a prime $p$ such that $p^{2}$ does not divide $\left|\mathrm{A}_{n}\right|$. As it is easy to check, using [CCNPW], the remaining simple groups do not satisfy the above condition either.) I think that each group satisfying the above condition has a nontrivial abelian normal subgroup. If $\chi(1)^{2}$ divides $|G / \operatorname{ker}(\chi)|$ for all $\chi \in \operatorname{Irr}(G)$, it is possible to prove that $G$ is solvable, using the classification of finite simple groups and working by induction. After completing this note, I.M. Isaacs informed me that S. Gagola and M. Lewis have proved that, in fact, the group under consideration is nilpotent; this yields a new characterization of nilpotent groups.

Corollary 6 (Compare to [BZ], Theorem 9.16). Let $G$ be a nonabelian group and $p$ a prime divisor of $|G|$. Then the following assertions are equivalent:

(a) For any nonlinear irreducible character $\chi$ of $G$, there exists $s=s_{\chi} \in \mathbb{N}$ such that $|G / \operatorname{ker}(\chi)|=p^{s} \cdot \chi(1)^{2}$.

(b) $G$ is a p-group.

Proposition 7. Let $G$ be a nonabelian group.

(a) Suppose that, for every monolithic character $\chi$ of $G$, there are a prime $p=p_{\chi}$ and an integer $\epsilon=\epsilon_{\chi} \in\{0,1\}$ such that $|G / Z(\chi)|=p^{\epsilon} \cdot \chi(1)^{2}$. Then $G$ is nilpotent.

(b) Suppose that, for any nonlinear monolithic character $\chi$ of $G$, there is a prime $p=p_{\chi}$ such that $|G / \operatorname{ker}(\chi)|=p \cdot \chi(1)^{2}$. Then $G$ is nilpotent. Furthermore, if the above condition holds for all nonlinear $\chi \in \operatorname{Irr}(G)$, then $G$ is a prime power group.

Proof. (a) We will use induction on $|G|$. Let $H$ be a minimal normal subgroup of $G$. By the inductive hypothesis, $G / H$ is nilpotent. Therefore, we may assume that $H$ is the unique minimal normal subgroup of $G$. In that case, $G$ possesses a faithful irreducible character $\chi$; obviously, $\chi$ is monolithic. Since $G$ is not nilpotent, $\mathrm{Z}(G)=\{1\}$ so $\mathrm{Z}(\chi)=\{1\}$ and $|G|=p \cdot \chi(1)^{2}$, where $p$ depends on $\chi$. We see that $|G|_{q}$ is not a square for exactly one $q \in \pi(G)$ (obviously, $q$ must be equal to $p$ ). Therefore, if $\theta$ is another faithful irreducible character of $G$, then $|G|=p \cdot \theta(1)^{2}$ with the same $p$ so $\theta(1)=\chi(1)$, i.e., all faithful irreducible characters of $G$ have the same degree $\chi(1)$. If $q \in \pi(G)-\{p\}$ and a nonlinear $\tau \in \operatorname{Irr}(G)$ is such that $q \nmid \tau(1)$, then, by the above, $\tau$ is not faithful; it follows that $H \leq \operatorname{ker}(\tau)$ and so $H \leq G\left(q^{\prime}\right)$. It follows that $H$ is solvable, by Theorem 2(b). Let $H$ be a $q$-group. Assume that $G$ is not nilpotent. Then $H \not \leq \Phi(G)$, by $(\Phi 1)$, so $G=M \cdot H$, where 
$M$ is maximal in $G$. Since $H$ is unique and $M$ is nilpotent, $H \in \operatorname{Syl}_{q}(G)$. By Ito's Theorem, $q$ does not divide $\chi(1)$. In that case, by hypothesis, $|H|=q$ so $p_{\chi}=q$. Since $\mathrm{C}_{G}(H)=H, G / H$ is cyclic, and $G$ is a Frobenius group so $\chi(1)=|G / H|$, by [I], Theorem 6.34. As $\chi(1)^{2}$ does not divide $|G|$, we get a contradiction.

(b) The first assertion follows from (a) immediately. The second assertion follows from Corollary 6.

In particular, $|G / \mathrm{Z}(\chi)|=\chi(1)^{2}$ for all $\chi \in \operatorname{Irr}(G)$ if and only if $G$ is nilpotent and all its Sylow subgroups satisfy the same condition (this also follows from Lemma 4). Probably, the derived length of such $G$ is bounded.

There is no nonabelian $p$-group $G$ such that $|G / \mathrm{Z}(\chi)|=p \cdot \chi(1)^{2}$ for all nonlinear $\chi \in \operatorname{Irr}(G)$; indeed, the $p$-groups $G$ with $\left|G^{\prime}\right|=p$ do not satisfy this condition. Therefore, in Proposition $7(\mathrm{a}), \epsilon \in\{0,1\}$. This example also explains why a $p$ group $G$ is abelian if $\chi(1)^{3}$ divides $|G / \mathrm{Z}(\chi)|$ for all irreducible characters $\chi$ of $G$.

Proposition 8. Let $G$ be a nonabelian solvable group.

(a) Suppose that, for any nonlinear monolithic character $\chi$ of $G$, there is a square free number $t_{\chi} \in \mathbb{N}$ such that $|G / Z(\chi)|=t_{\chi} \cdot \chi(1)^{2}\left(|G / \operatorname{ker}(\chi)|=t_{\chi} \cdot \chi(1)^{2}\right)$. Then $G$ is nilpotent.

(b) Let, in addition, $G$ is of odd order. Suppose that, for any monolithic character $\chi$ of $G$, there is a cube free number $t_{\chi}$ such that $|G / Z(\chi)|=t_{\chi} \cdot \chi(1)^{2}$. Then $G$ is nilpotent.

Proof. Working by induction on $|G|$, we may assume that $G$ has only one minimal normal subgroup $H$, and $G / H$ is nilpotent. Assuming that $G$ is not nilpotent, we have $\mathrm{Z}(G)=\{1\}$ and $H \not \leq \Phi(G)$.

If $\chi \in \operatorname{Irr}(G)$ is faithful (such a $\chi$ exists since $G$ is a monolith), then $|G|=$ $t_{\chi} \cdot \chi(1)^{2}$, where $t_{\chi}$ is square free in (a) and cube free in (b). Let $M$ be a maximal subgroup of $G$ such that $H \not \leq M$. Then $G=M \cdot H$, a semidirect product. Since $H$ is unique, $M_{G}=\bigcap_{x \in G} M^{x}=\{1\}$. In that case, $H \in \operatorname{Syl}_{p}(G)$ for some prime $p$, and this implies that $|H|=p$ or $p^{2}$, by Ito's Theorem. It follows that $\mathrm{C}_{G}(H)=H$ so $M$ is a nilpotent $p^{\prime}$-subgroup of $\mathrm{GL}(1, p)$ or $\mathrm{GL}(2, p)$.

In case (a), $G$ is a Frobenius group; then $\chi(1)^{2}$ does not divide $|G|$ (see [I], Theorem 6.34), which is a contradiction.

In case (b), since $M$ is a nilpotent $p^{\prime}$-subgroup of odd order in $\operatorname{GL}(n, p), n \leq 2$, it follows that $M$ is abelian. So, if $x \in M^{\#}$, then $\mathrm{C}_{G}(x)=M$ since $M_{G}=\{1\}$. It follows that $G$ is a Frobenius group. If $\chi$ is a faithful irreducible character of $G$, then $\chi(1)=|G: H|$, by $[\mathbf{I}]$, Theorem 6.34 , and so $\chi(1)^{2}$ does not divide $|G|$, a contradiction.

Probably, Proposition 8(a) is true without assumption on solvability of $G$ (I think that the proof of this must depend on the classification of finite simple groups).

The group $G$ of the Chillag-Herzog Theorem (see [BZ], Theorem 14.31) possesses, as a rule, a few irreducible character degrees. In the opposite direction, I'd like to pose the following.

Problem 1. Let $G$ be a nonabelian group such that, for every divisor $m$ of its order such that $m^{2}<|G|, G$ possesses an irreducible character of degree $m$. Describe the structure of $G$. Is it true that $G^{\prime}<G$ ? 
Let $G$ be as in Problem 1 and $|G|$ is divisible by $p^{2}$. Then, by [BZ, Corollary $3.23, \mathrm{Z}(\chi)>\{1\}$ for every irreducible character $\chi$ of degree $p$. In spite of the set of these groups being very small, their classification is surprisingly difficult.

Problem 2. Suppose that for every nonlinear irreducible character $\chi$ of $G$, one has $|\pi(G / Z(\chi))-\pi(\chi(1))| \leq 1$. Describe the structure of $G$.

For related results, see [BZ], Chapters 9, 14, 30, and [BIK].

I am indebted to the referee for numerous remarks and suggestions which improved this note considerably.

\section{REFERENCES}

[B] Y. Berkovich, On Isaacs' three character degrees theorem, Proc. Amer. Math. Soc. 125, 3 (1997), 669-677. MR 97i:20006

[BIK] Y. Berkovich, I. M. Isaacs and L. Kazarin, Distinct monolithic character degrees, J. Algebra 216 (1999), 448-480.

[BZ] Y. G. Berkovich and E.M. Zhmud', Characters of Finite Groups. Parts 1, 2, Translations of Mathematical Monographs 172, 181, American Mathematical Society, Providence, 1998. MR 98m:20011

[CCNPW] J. H. Conway, R. T. Curtis, S. P. Norton, R. A. Parker, R. A. Wilson, Atlas of Finite Groups, Clarendon Press, Oxford, 1985. MR 88g:20025

[I] I.M. Isaacs, Character Theory of Finite Groups, Academic Press, New York, 1976. MR 57:417

[W] W. Willems, Blocks of defect zero in finite simple groups, J. of Algebra 113 (1988), 511-522. MR 89c:20025

Department of Mathematics, University of Haifa, Mount Carmel, Haifa 31905, Israel

E-mail address: berkov@mathcs2.haifa.ac.il 